\title{
Effect of anterior strut chordal transection on the force distribution on the marginal chordae of the mitral valve
}

\author{
Muralidhar Padala, PhD, ${ }^{a}$ Lazarina Gyoneva, BS, ${ }^{\mathrm{b}}$ and Ajit P. Yoganathan, $\mathrm{PhD}^{\mathrm{b}}$
}

\begin{abstract}
Objectives: Transection of the secondary chordae on the anterior leaflet of the mitral valve to relieve leaflet tethering and reduce regurgitation is an experimentally proven procedure to correct functional mitral regurgitation. In the present study, we sought to investigate whether transecting the secondary chordae would have an effect on the marginal chordal force on the same leaflet.
\end{abstract}

\begin{abstract}
Methods: Adult porcine mitral valves $(\mathrm{n}=8)$ were studied in a pulsatile heart simulator, in which the papillary muscle positions can be precisely positioned. Miniature transducers were inserted into the anterior marginal chordae to measure the chordal forces. Each valve was studied under baseline conditions, 3 different tethering conditions (apical, apical-lateral, and apical-lateral-posterior), and after chordal cutting in the 3 tethering conditions. The temporal changes and peak and average marginal chordal forces under each condition are reported.
\end{abstract}

\begin{abstract}
Results: Apical tethering increased the marginal chordal force by an average of $96 \%$ but remained unchanged after chordal cutting. With apical-lateral tethering, the marginal chordal force increased by $210 \%$ from baseline and increased further to $350 \%$ of baseline after chordal cutting. After apical-lateral-posterior tethering, the marginal chordal force increased to $335 \%$ of baseline before transection and by $548 \%$ after transection.
\end{abstract}

Conclusions: The increase in the marginal chordal force after secondary chordal cutting depends on the location of the papillary muscles and the extent of leaflet tethering. Although chordal cutting might not alter the valve mechanics under minimal leaflet tethering, it significantly affects the mechanics when the leaflet tethering is more pronounced, which is typically seen in patients with functional mitral regurgitation. (J Thorac Cardiovasc Surg 2012;144:624-33)

Supplemental material is available online.

Functional mitral regurgitation (MR) is a secondary pathologic finding in patients with dilated remodeled ventricles resulting from ischemic or nonischemic etiologies. ${ }^{1,2}$ Ventricular dilation-induced papillary muscle displacement results in apical tethering of the anterior and posterior leaflets, resulting in MR. Mitral annuloplasty is currently used to repair MR by reducing the septal-lateral dimension of the

From the Division of Cardiothoracic Surgery, ${ }^{\mathrm{a}}$ Emory University School of Medicine, Atlanta, Ga; and Wallace H. Coulter Department of Biomedical Engineering, ${ }^{b}$ Georgia Institute of Technology, Atlanta, Ga.

M. Padala was supported by an American Heart Association Fellowship (grant 0815159E) during the course of this study at Georgia Institute of Technology, L. Gyoneva was supported by a Georgia Tech Presidential Undergraduate Fellowship, and A. P. Yoganathan and the research work were partially supported by National Heart, Lung, and Blood Institute grant R01-HL090661.

Disclosures: Authors have nothing to disclose with regard to commercial support.

Received for publication May 27, 2011; revisions received Sept 12, 2011; accepted for publication Oct 20, 2011; available ahead of print Dec 9, 2011.

Address for reprints: Ajit P. Yoganathan, PhD, Wallace H. Coulter Department of Biomedical Engineering, Georgia Institute of Technology, Atlanta, GA (E-mail: ajit.yoganathan@bme.gatech.edu).

$0022-5223 / \$ 36.00$

Copyright (c) 2012 by The American Association for Thoracic Surgery doi:10.1016/j.jtcvs.2011.10.032 mitral annulus. However, apical tethering of the leaflets persists after this procedure and is known to contribute to persistent or recurrent regurgitation. ${ }^{3,4}$ Pronounced concavity of the anterior leaflet (seagull shape) is vivid in these patients both before and after annuloplasty and is associated with greater degrees of recurrence of the regurgitation. ${ }^{5}$ The anterior strut chordae of the mitral valve insert at the region of maximum leaflet bending and have been implicated in tethering the entire anterior leaflet surface between their insertion sites and the mitral annulus (Figure 1).

Transection of the anterior strut chordae to relieve anterior leaflet tethering was proposed as an adjunct technique to annuloplasty to correct MR and improve leaflet coaptation in these patients. ${ }^{6-8}$ Although clinical experience with this procedure is limited, the hemodynamic efficacy of this technique in chronic ischemic animal models has been documented, and its potential role in diminishing left ventricular remodeling is emerging. ${ }^{9}$ Favorable experimental results have encouraged clinical adoption of a version of this procedure by Borger and colleagues ${ }^{10}$ that involved cutting several chordal tendineae; however, the effect of transecting the anterior strut chordae, as proposed by Messas and colleagues ${ }^{8}$ on mitral valve mechanics is in question. Specifically, the effect of anterior strut chordal cutting on the thinner marginal chordae on the anterior leaflet in a disease state is not fully understood. 


\section{Abbreviation and Acronym}

$\mathrm{MR}=$ mitral regurgitation

In a previous study, our group measured the chordal force distribution under different mitral valve tethering settings and reported that the anterior strut chordae of the mitral valve sustained the maximum loads during systole, and the anterior marginal chordae sustained loads an order of magnitude less than did the strut chordae. ${ }^{11}$ Subsequently, we also demonstrated that the strut chordae were structurally robust, with a thick collagen core that is encapsulated in an elastin layer, and were highly extensible compared with the marginal chordae, which were thin and least extensible compared with the other chordae. ${ }^{12} \mathrm{He}$ and colleagues ${ }^{13,14}$ recently reported the effect of anterior strut chordal cutting on the marginal chordal force in an ex vivo static pressurized heart model and demonstrated that significant changes in anterior marginal chordal force were observed only when the strut chord from the same papillary muscle was transected. However, considering that their model was limited to a static, nondilated pressurized heart, the investigators noted the relevance of their study to the setting of chordal rupture and not to functional MR. In a similar model, Chen and MayNewman ${ }^{15}$ measured the changes in leaflet strain after chordal cutting under normal conditions and reported an increase in radial strain. However, to enable clinical translation of ex vivo mechanics measurements, a model that mimics the pulsatile hemodynamics and annular and subannular geometric distortions relevant to a dilated ischemic ventricle is necessary. In the present study, using a novel pulsatile mitral valve model, we sought to investigate the changes in the anterior marginal chordal forces after chordal cutting under clinically relevant geometric valve distortions and pulsatile hemodynamic conditions.

\section{MATERIALS AND METHODS \\ Experimental Model}

A pulsatile left heart simulator is shown in Figure 2, A. It allows precise dilation of the mitral annulus, precise positioning of the papillary muscles in 3-dimensional space and quantitative and visual measurements on the valve. This model was previously reported for investigation of the integrated mechanisms of MR,,$^{1,12,16}$ to study the changes in chordal force balance with papillary muscle position, ${ }^{11}$ and to study the efficacy of chordal cutting to relieve anterior leaflet tenting. ${ }^{4}$ More recently, it was also validated against high temporal and spatial in vivo leaflet strain measurements. ${ }^{17}$ In this model, instead of using a contractile and complex ventricular myocardial model, we used geared mechanisms that can precisely alter the papillary muscle position to mimic adverse ventricular remodeling. A saddle-shaped mitral annulus made of flexible silicone tubing, whose area/size can be altered using a spring mechanism, was used.

\section{Mitral Valve Extraction and Preparation}

Fresh porcine mitral valves were used because of their anatomic similarity to human mitral valves and to be consistent with in vivo reports from other laboratories that used Yorkshire pigs. The use of normal human mitral valves would be ideal, but it is challenging to obtain a sufficient number of fresh transplant hearts for research purposes at our institution. Fresh pig hearts $(\sim 50-60-\mathrm{kg}$ pigs) were obtained from a local slaughter house (Hollifield Farms, Covington, Ga) immediately after death and were transported to the laboratory in ice-cold saline. After removing the pericardium and excising the left atrium, mitral valve sizing was performed by pressurizing the heart to $120 \mathrm{~mm} \mathrm{Hg}$. The anterior leaflet size was measured using an Edwards Lifesciences Physio ring sizer (Figure 2, B), and the $28-\mathrm{mm}$ valves were selected. Two-dimensional echocardiography was performed to measure the distance of the papillary muscle tips from the mitral annulus in each valve and recorded (Figure 2, C). The valves were extracted from the hearts, leaving $5 \mathrm{~mm}$ of atrial tissue proximal to the mitral annulus intact, and preserving the entire leaflet, chordal, and papillary muscle apparatus (Figure 2,D).

\section{Experimental Preparation}

The extracted mitral valves were mounted into the pulsatile simulator by suturing the left atrial tissue onto the silicone annulus in a mattress fashion

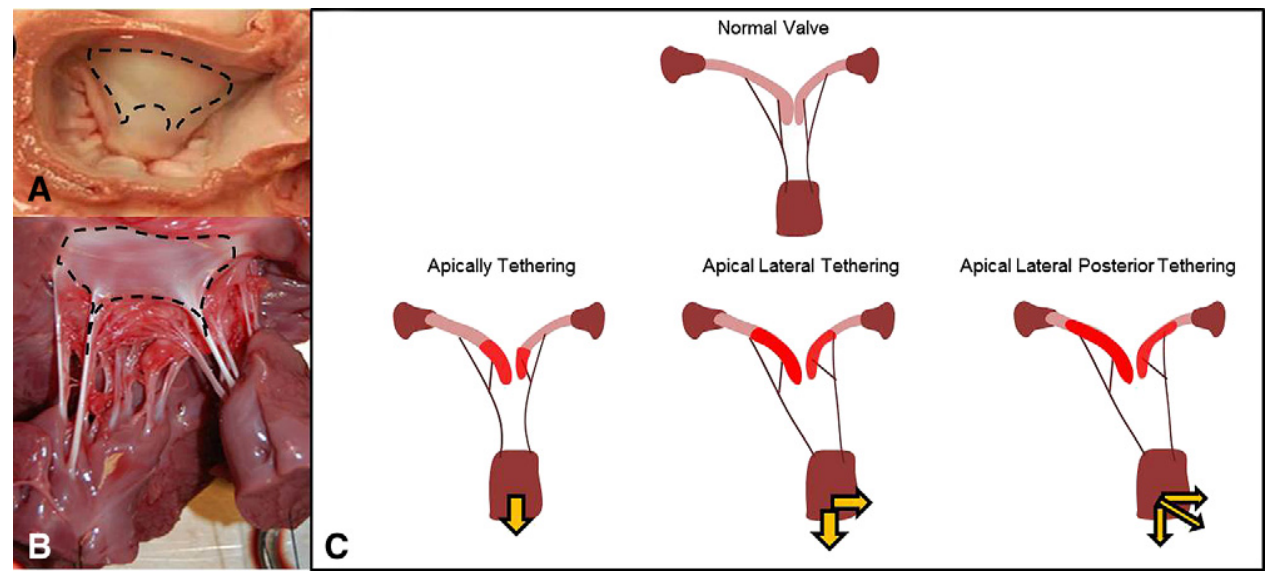

FIGURE 1. A, Atrial surface of anterior leaflet with collagen fibers inserting from anterior strut chordae tendineae; B, ventricular surface of anterior leaflet with fibers from strut chordae; and C, different settings of anterior leaflet tethering for variety of papillary muscle positions. 


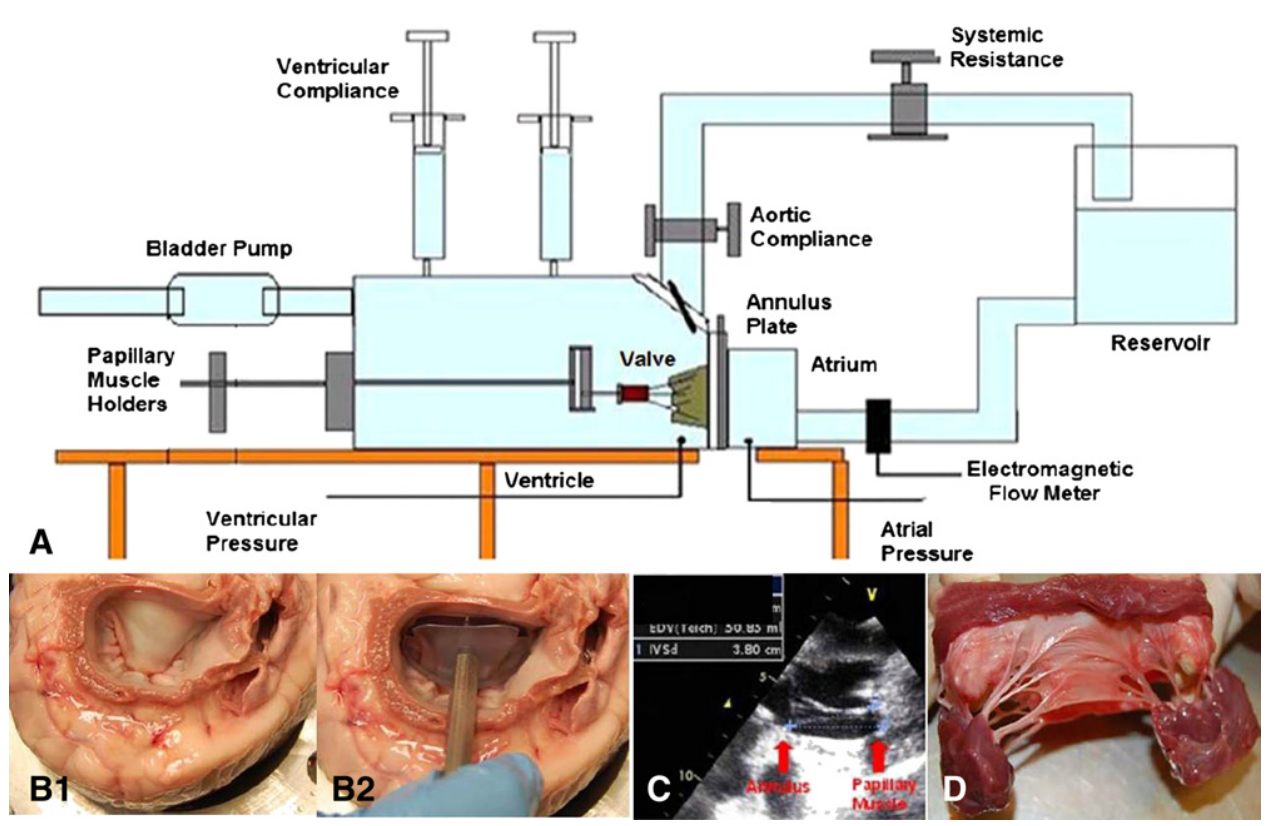

FIGURE 2. A, Ex vivo pulsatile left heart simulator in which chordal forces on native mitral valve chordae tendineae can be measured. B, Mitral valve leaflet and annular sizing using a sizer. C, In vivo measurement of annular tip to papillary muscle tip distance before explanting mitral valve. D, Explanted mitral valve with all structures intact.

(Figure 3, A) and mounting the papillary muscles onto the geared papillary muscle holders (Figure 3, $B$ ). The silicone annulus can dilated from $28 \mathrm{~mm}$ to $40 \mathrm{~mm}$, and the papillary muscle holders can be displaced to a resolution of $0.9 \mathrm{~mm}$ in the apical, posterior, and lateral directions. On mounting the valve in the simulator, a solenoid-timed bulb pump was attached to the ventricle, and desired pressure and flow waveforms were generated.

\section{Chordal Force Measurement}

Two marginal chordae on the anterior leaflet then had miniature C-ring transducers inserted (Figure 3,D). We had previously used these with success to measure the mitral valve chordal forces. These C-ring force transducers consist of 2 strain gauges, 1 on the convex and 1 on the concave side of the ring structure. By connecting the 2 gauges in a half bridge circuit, the voltage drop across them can be measured. The voltage drop across the transducers was calibrated against measured loads before the experiment. Continuous hemodynamic monitoring was performed using differential pressure transducers (DP-09-40; Validyne Engineering, Northridge, Calif) and an electromagnetic flow probe (600 Model, 501D Analog Meter; Carolina Medical Electronics, East Bend, NC) throughout the experiment. Continuous data monitoring and recording were performed using
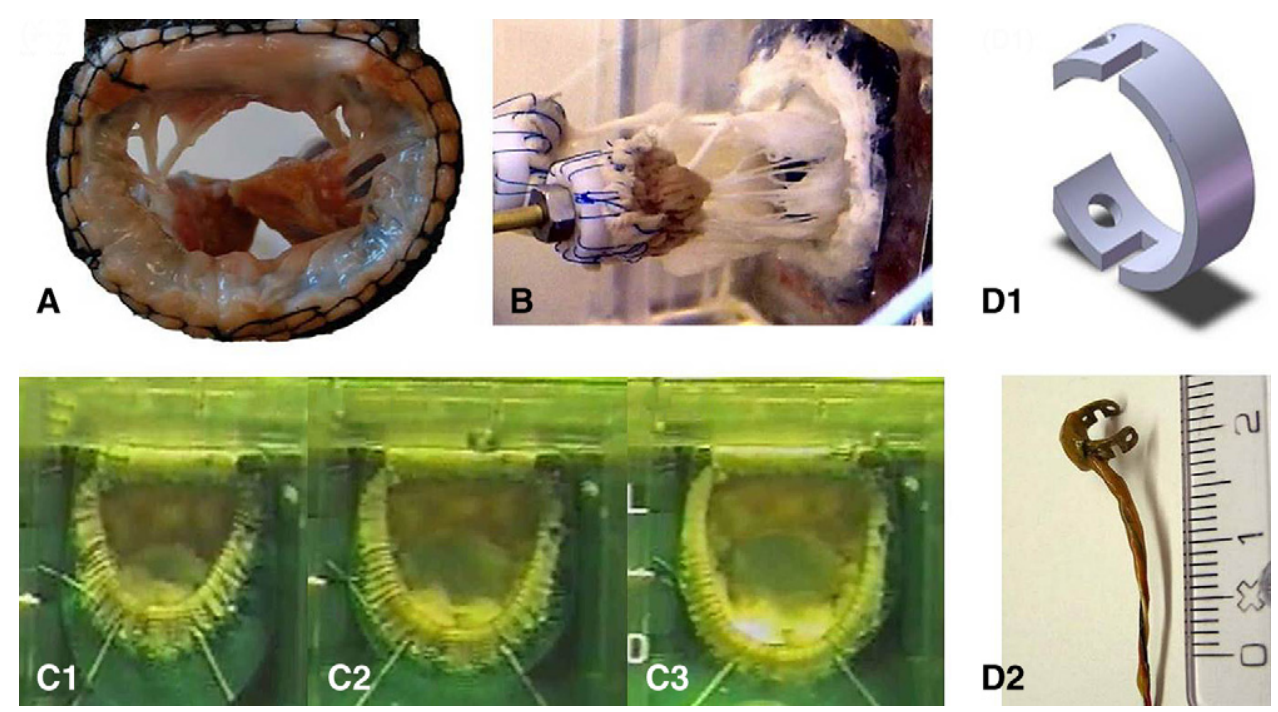

FIGURE 3. A, Extracted mitral valve sutured onto silicone annulus using sutures. B, Mitral valve mounted into left heart simulator. C, Annular size increase induced with silicone annulus to simulate mitral annular dilation. D, Miniature C-ring force transducers developed with strain gauge technology to mount on chordae tendineae to measure forces acting on them throughout cardiac cycle. 

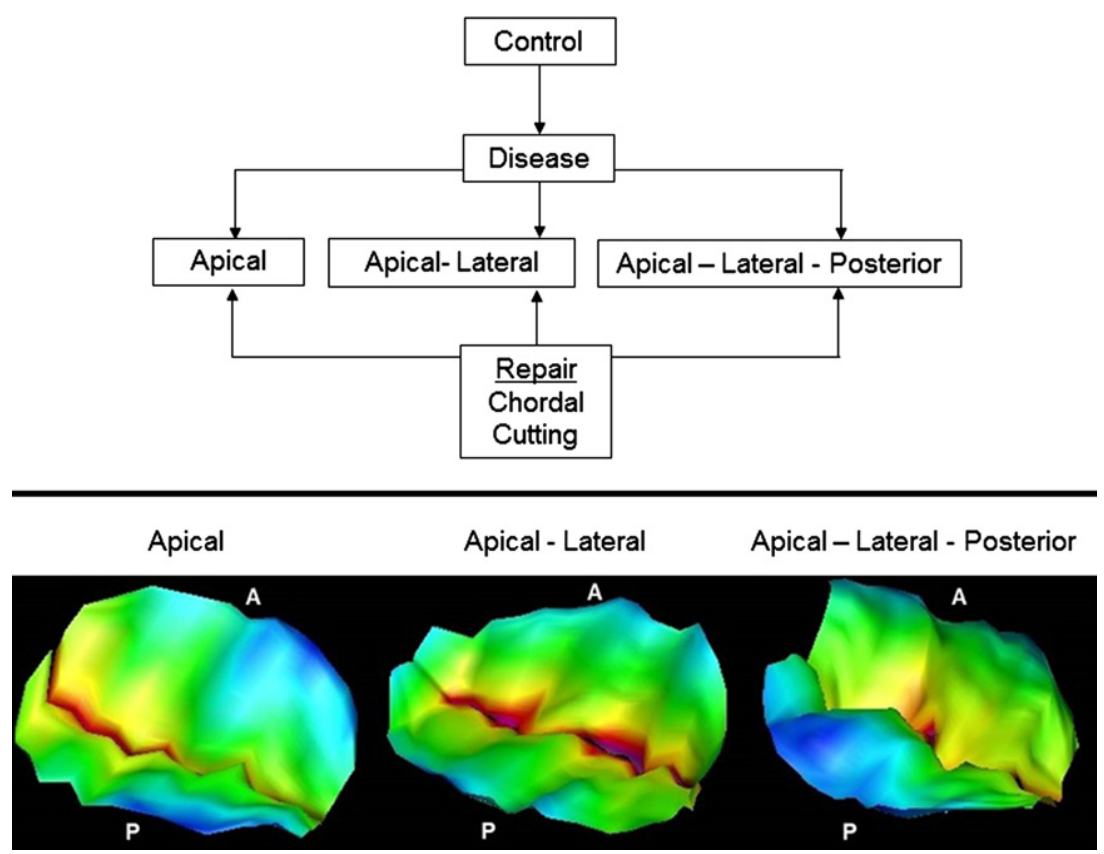

FIGURE 4. Top, Experimental protocol used in our study. Bottom, Representative images of mitral valve tethering obtained under different pathologic mitral valve papillary muscle positions, reconstructed from 3-dimensional echocardiograms.

a high-frequency data acquisition system (National Instruments PCMCIA DAQCard-1200, Austin, Tex), and data acquisition was timed to the cardiac cycle using a synchronizing signal.

\section{Imaging Protocol}

Gated echocardiographic acquisitions were performed using a 2-dimensional adult probe (Vivid 7; GE Healthcare, Waukesha, Wis) for all the experiments. A 3-dimensional matrix array probe (Philips iE33; Philips Medical Systems, Andover, Mass) was used in 1 experiment owing to the limited availability of a 3-dimensional ultrasound machine for research purposes at our institution. All images were acquired at $3.75 \mathrm{MHz}$, at an imaging depth of $12 \mathrm{~mm}$, and the echocardiographic images were acquired along the septal-lateral axis at the central and commissural cusps.

\section{PROTOCOL}

\section{Experimental Protocol}

The experimental protocol for each valve in the present study is summarized in Figure 4, A.

Control/baseline conditions. The annulus was maintained at a $28-\mathrm{mm}$ physiologic size, and the papillary muscles were in their physiologic state (as determined by the measurements made in the pressurized hearts). Each valve was studied under pulsatile hemodynamic conditions of $120 \mathrm{mmHg}$ peak transmitral pressure and $5 \mathrm{~L} / \mathrm{min}$ cardiac output at 70 beats per minute.

Functional mitral regurgitation conditions. The annulus was dilated to $36 \mathrm{~mm}$ by increasing the silicone annular size (Figure 3,C). The papillary muscles were displaced by 10 $\mathrm{mm}$ in the apical direction (group 1) and the measurements taken, followed by an additional $10 \mathrm{~mm}$ in the lateral direction (group 2), and an additional $10 \mathrm{~mm}$ in the posterior direction (group 3). The order of simulating these displacements was randomized among the different valves to avoid any systematic error resulting from the procedure.

Anterior strut chordal cutting. After confirmation of leaflet tethering on ultrasonography, the anterior strut chordae were transected using a miniature catheter that could be inserted into the experimental setup without disturbing the mitral valve. The insertion point of the chordae into the leaflet was isolated using a snare, and the chord was transected at the insertion point by taking care that no damage to the leaflet was made, which was ensured by visual examination.

\section{Measurement Techniques}

Chordal force measurements. Forces on the marginal chordae were measured for 15 consecutive cardiac cycles in each experimental condition, and the averaged temporal data and peak force magnitudes are reported.

Factor of safety. The factor of safety was calculated as the ratio of the average peak force measured on the marginal chordae in the present study and the failure strength reported by Sedransk and colleagues ${ }^{18}$ from uniaxial testing. This parameter can be useful in assessing the safety from rupture of the marginal chord under different experimental conditions.

\section{Statistical Analysis}

All measured endpoints are reported as the mean \pm standard deviation. The data were tested for normality using the Anderson-Darling test in Minitab, version 15 (Minitab, State College, $\mathrm{Pa}$ ). The experimental groups were compared 


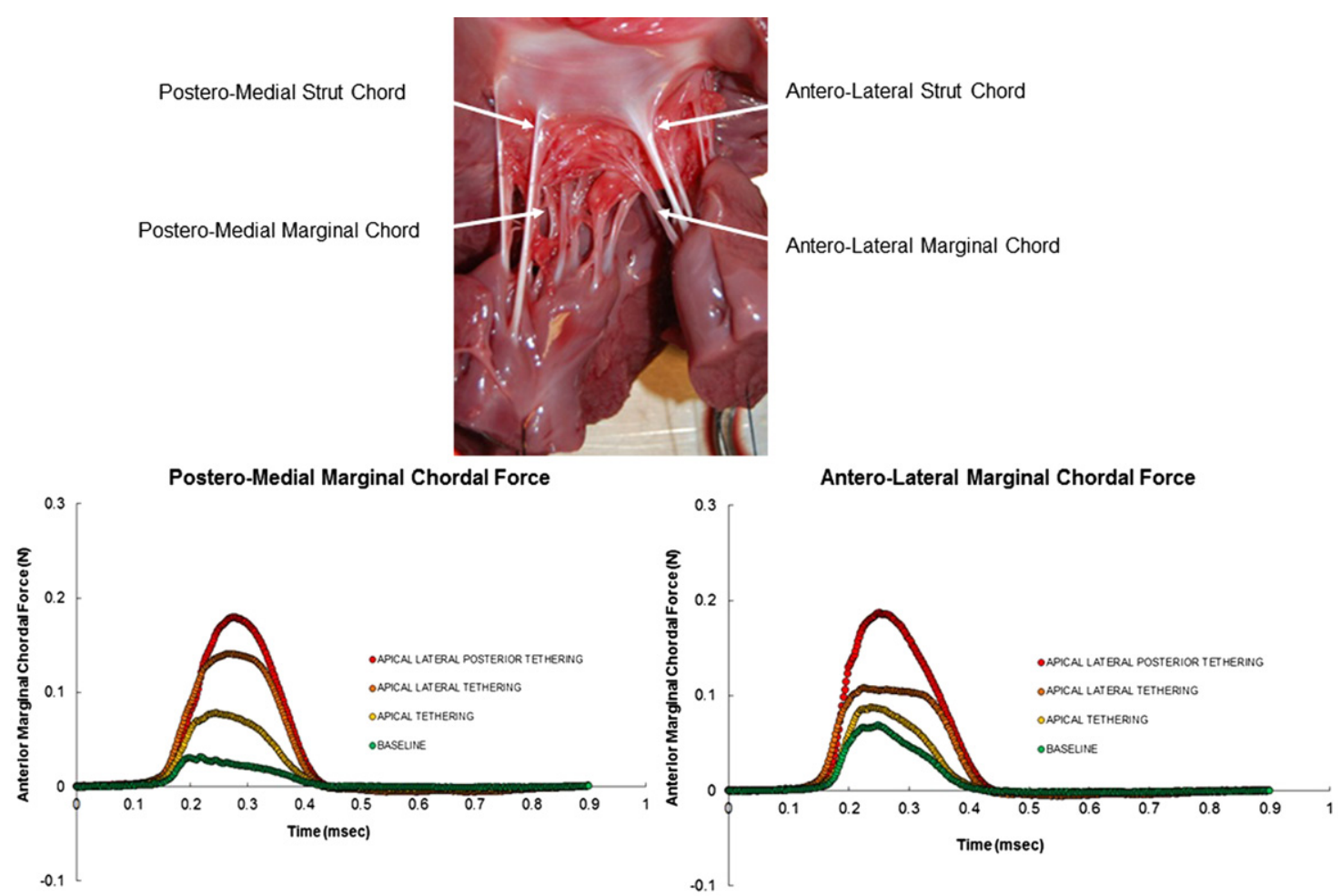

FIGURE 5. Top, Ventricular surface of mitral anterior leaflet depicting insertion sites of strut and marginal chordae. Bottom, Left, Posteromedial papillary muscle marginal chordal force at normal and different pathologic papillary muscle positions before chordal cutting. Bottom, Right, Anterolateral papillary muscle marginal chordal force at normal and different pathologic papillary muscle positions before chordal cutting.

using a paired $t$ test, with statistically significant differences at $P<.05$, with the $95 \%$ confidence intervals. A nonparametric Wilcoxon signed-rank test was conducted on the leaflet when the data were not normally distributed.

\section{RESULTS \\ Leaflet Tethering Pattern}

To establish that the desired tethering pattern was achieved and that it was different among the 3 displacement directions, the leaflet shape was segmented from the echocardiograms, and the tenting distance from the mitral annular plane was mapped (Figure 4, B). With apical displacement, the tethering was concentrated at the free edges of the 2 leaflets. With apical-lateral displacement, the tethering was more pronounced but still focused near the free edges. Finally with apical-lateral-posterior displacement, the tethering was diffused over the 2 leaflets but was significantly greater on the anterior leaflet, producing the typical "seagull" shape on 1 leaflet and a slight prolapse on the other leaflet.

\section{Temporal Marginal Chordal Forces Before Chordal Cutting}

Figure 5 depicts the temporal changes in the marginal chordal forces (left and right anterior marginal chordae) under different tethering patterns and compared with baseline. At baseline, a force trace that increased rapidly with early systole, plateaued relatively during early to midsystole, and gradually reduced during late systole; similar to the characteristic force measurements reported by Salisbury and colleagues ${ }^{19}$ in an animal model, but on the secondary chordae. With apical displacement of the papillary muscles, an increase in the peak force was measured, but the temporal trend remained similar to that at baseline, if not identical. When the papillary muscles were moved laterally, the early systolic increase in force was identical, but a rather flat plateau was observed for rest of systole, followed by a rapid decline at end systole. With additional posterior displacement of the papillary muscles, the plateau was eliminated, with a more or less curved peak in the force that involved a rapid increase to peak and then a rapid decline.

\section{Temporal Marginal Chordal Forces After Chordal Cutting}

Figure 6 shows a comparison between the marginal chordal force before and after chordal cutting under each of the papillary muscle positions for the left and right marginal chordae. After chordal cutting, the gradual decline or the plateau seen before chordal cutting was lost, with a rapid 

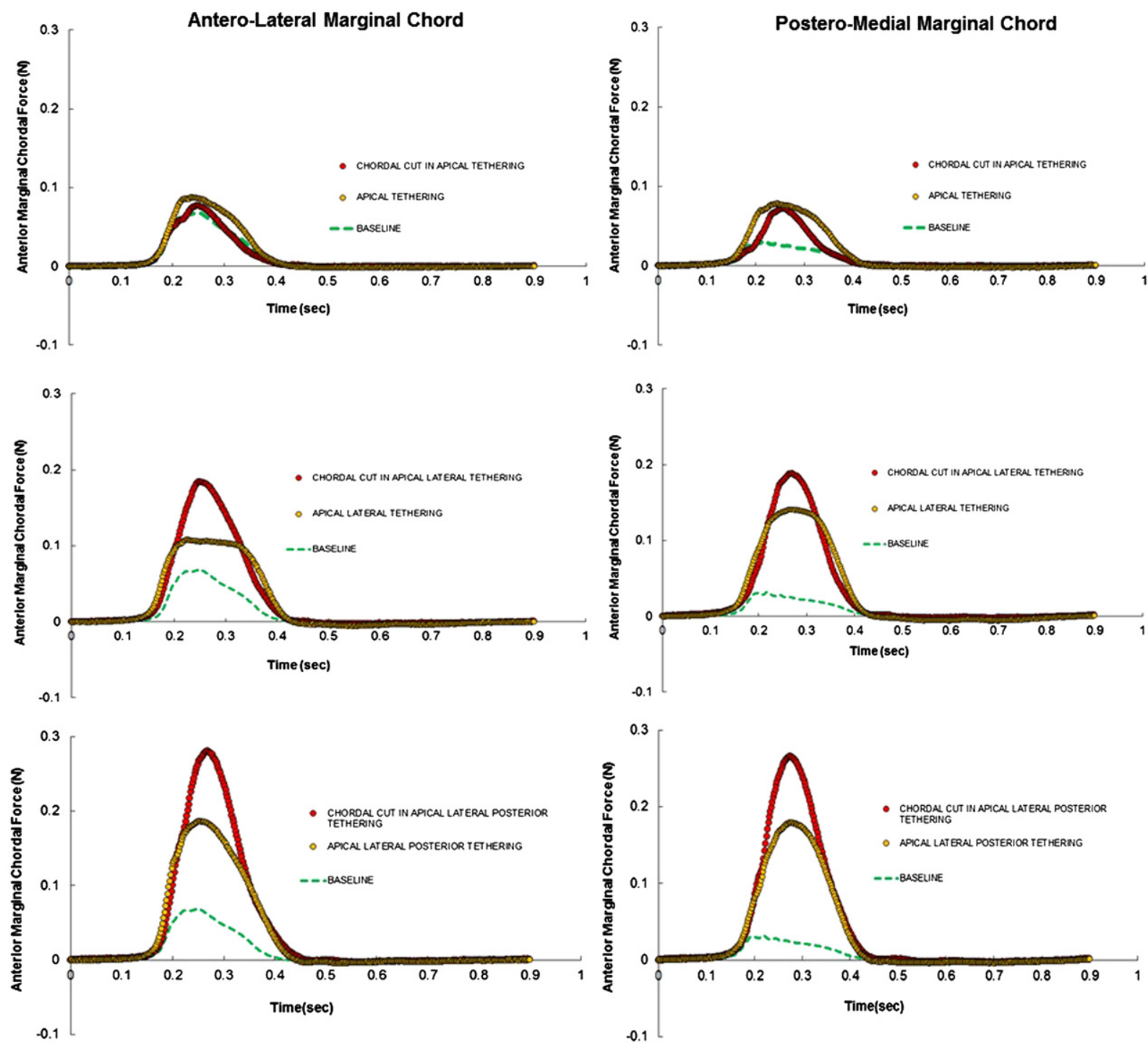

FIGURE 6. Changes in marginal chordal forces before and after chordal cutting in left and right marginal chordae under different papillary muscle positions. Row 1, Apical papillary muscle displacement depicting minimal changes in measured chordal forces before and after chordal cutting. Row 2, Apical lateral papillary muscle displacement depicting significant increase in chordal force before and after chordal cutting in both chordae. Row 3, Apical lateral posterior papillary muscle displacement depicting significant changes in chordal force before and after chordal cutting in both chordae.

increase to peak, followed by a rapid decrease in the force during systole. Compared with the force trace measured before chordal cutting, an increase in the peak force was observed only with apical-lateral $(P=.04)$ and apicallateral-posterior $(P=.05)$ displacement, but not with apical displacement $(P=.86)$. The potential mechanisms of such change compared with those recorded before chordal cutting are detailed in subsequent sections.

\section{Peak Marginal Chordal Forces}

The peak forces measured in each setting, the percentage of change compared with baseline and before chordal cutting, and an overall factor of safety are listed in Table 1.
In the left marginal chord, a $45 \%$ increase in peak force was measured after apical displacement compared with baseline that, after chordal cutting, actually decreased to $17 \%$. With apical-lateral displacement, an $80 \%$ increase in force was measured before chordal cutting that increased to $208 \%$ after chordal cutting procedure. After apicallateral-posterior displacement, a $210 \%$ increase in force was measured before chordal cutting that subsequently increased to $367 \%$ after chordal cutting. In the right marginal chord, the increase in forces followed a similar trend but with greater magnitude changes. A $143 \%$ increase in peak force was measured after apical displacement compared with baseline that after chordal cutting actually 
decreased to $119 \%$. With apical-lateral displacement, a $341 \%$ increase in force was measured before chordal cutting that increased to $491 \%$ after chordal cutting. After apical-lateral-posterior displacement, a $459 \%$ increase in force was measured before chordal cutting that subsequently increased to $728 \%$ after chordal cutting.

\section{DISCUSSION}

Anterior strut chordal transection is a hemodynamically proven technique to relieve MR in an ovine model of acute and chronic ischemia. ${ }^{7,8}$ In a dilated ventricle, the strut chordae significantly tether the anterior leaflet, and their transection relieves the leaflet tethering and allows better basal motion toward the mitral annulus and lateral motion toward the posterior leaflet. Although this is a hemodynamically and clinically appealing mechanism to restore valve competence in a tethered mitral valve, surgeons have been skeptical about implementing this procedure in humans owing to the notion that the smaller chordae tendineae might experience significantly high forces after the procedure. In the present study, we attempted to provide a quantitative measure of the increase in the marginal chordal force compared with the failure strength of the anterior marginal chord.

In general, the data from our study have demonstrated that anterior strut chordal cutting increases the forces on the anterior marginal chordae in a papillary muscle position-dependent manner. First, even before chordal cutting, significant increases in the marginal chordal force were measured, dependent on the location of the papillary muscles in the left ventricle. After chordal cutting, no statistically significant increases in the marginal chordal force were measured when the papillary muscles were apically displaced. However, with apical-lateral and apical-lateralposterior displacement, the increase in the marginal chordal force was significant both before and after chordal cutting compared with baseline.

The baseline marginal chordal forces measured in the present study were within the range previously reported by Salisbury and colleagues, ${ }^{19}$ Nielsen and colleagues, ${ }^{20,21}$ and Jimenez and colleagues, ${ }^{11}$ demonstrating the experimental accuracy of the techniques used in the present study. $\mathrm{He}$ and colleagues ${ }^{13}$ in an isolated pressurized heart previously demonstrated that transection of the strut chordae significantly increases the marginal chordal tension. Their study demonstrated that the anterior marginal chordal force increased only when the anterior strut chord on the same leaflet was transected-speculating a chordal control system for each papillary muscle. These results seem realistic in the normal valve, because it was shown previously that the triangular segment encapsulated between the anterior marginal and strut chord (He's triangle) plays a role in the overall leaflet shape and forces on the chordae emanating from each papillary muscle head. However, because of the lack of papillary muscle displacement, as the investigators stated, their results would be more representative of a ruptured chordal setting than that of functional MR. In the present study, the measurement of forces in the marginal chord under different tethering conditions was used to provide quantitative evidence of the changes in marginal chordal forces after chordal cutting. In our study, apical displacement of the papillary muscles did not increase the marginal chordal force significantly, either before or after chordal cutting, although slight leaflet tethering was observed on the echocardiograms (Figure 4, $B$ ). We speculate that when chordal cutting relieves the slight tension that apical displacement imposes on the leaflet, the mobility of the leaflet is restored to physiologic levels. Thus, physiologic coaptation is restored, which literally mimics a native mitral valve, and thus explaining the minimal change in the chordal forces. With lateral displacement, it is reasonable to expect a significant increase in the leaflet free edge tension (see on-line Supplementary information) and an equivalent reduction in its mobility. Thus, the marginal chordal forces increase only to the point at which the free edge can move basally toward the mitral annulus, resulting in the force plateau, and then rapidly decrease as the leaflet is pulled/pushed back to its diastolic configuration. However, when the papillary muscles are displaced more posteriorly, the tethering would have increased to a large enough magnitude that the leaflet has reduced mobility in its entirety (as evident in Figure 4), resulting in incomplete coaptation and a regurgitation orifice (see Figures E1-E3). The leaflet thus tries to move basally toward the annulus, driven by the ventricular pressure, but the tethering forces overpower it and allow it to move only marginally such that it cannot meet with the posterior leaflet. Thus, an increase in the force is expected to reach a rapid peak, without a plateau, with, subsequently, a rapid decrease during late systole expected. These changes in the leaflet mobility in some of these settings were previously reported as the case of "leaflet loitering" by Glasson and colleagues. $^{22}$

The percentages of change in the marginal chordal forces from baseline and the factor of safety compared with the failure force of the chordae are listed in Table 1. The data indicate that as the distance of the papillary muscle tips from the mitral annulus increased, so did the force in the anterior marginal chordae tendineae before and after chordal cutting, with a significant decrease in the factor of safety. However, the measured forces were significantly smaller than the failure load of $6.8 \mathrm{~N}$ required for rupture of the anterior marginal chordae reported by Sedransk and colleagues. ${ }^{18}$ Although the forces measured in our study seem much smaller than the failure load, it is known that increased stretch in mitral valve tissue can accelerate remodeling and degeneration of the tissue and potentially lead to failure at much lower loading conditions than those 
TABLE 1. Maximum marginal chordal forces measured at different papillary muscle positions compared with baseline

\begin{tabular}{|c|c|c|c|c|c|c|c|c|}
\hline \multirow[b]{2}{*}{ Variable } & \multicolumn{4}{|c|}{$\begin{array}{c}\text { Average maximum marginal } \\
\text { chordal force before } \mathrm{CT} \text { cut }(\mathrm{n})\end{array}$} & \multicolumn{2}{|c|}{ Change from baseline (\%) } & \multicolumn{2}{|c|}{ Factor of safety } \\
\hline & Left & $P$ value & Right & $P$ value & Left & Right & Left & Right \\
\hline Apical PMD & $0.09 \pm 0.07$ & .37 & $0.08 \pm 0.1$ & .05 & 45 & 143 & 77.16 & 86.17 \\
\hline Apical lateral PMD & $0.127 \pm 0.05$ & .05 & $0.157 \pm 0.2$ & .05 & 80 & 340.6 & 61.96 & 47.22 \\
\hline Apical lateral posterior PMD & $0.216 \pm 0.12$ & .03 & $0.198 \pm 0.2$ & .05 & 210 & 459.3 & 35.55 & 36.98 \\
\hline
\end{tabular}

All $P$ values reported compared with baseline. Factor of safety is calculated as a ratio between the failure strength of the anterior marginal chord and the measured force in the experiments before and after chordal cutting: factor of safety $=$ [(failure force of anterior marginal chordae/measured force on anterior marginal chord) -1$]$. $C T$, chordal tendineae; $P M D$, papillary muscle displacement.

reported. Pedersen and colleagues, ${ }^{23}$ in an elegant study, demonstrated that porcine mitral valve leaflets subjected to chronic elevated load upregulated the expression of endothelin-B receptors, which are known to regulate key apoptotic pathways in degenerative mitral valve disease. Such degeneration might not be evident at surgery but can gradually increase, resulting in catastrophic failure of the repair at a later stage in the patient's life. We also observed that the increase in marginal chordal forces was consistently greater in the chordae from the posteromedial papillary muscle than in the anterolateral one in these porcine valves. Also, measurements of the chordal and papillary muscle length in pig hearts demonstrated that the chordae from the posteromedial muscle were consistently shorter than those from the anterolateral muscle (unpublished results). In the intact pig heart, the posteromedial papillary muscle is located on the inferior wall of the ventricle and closer to the mitral annular plane than is the anterolateral muscle, which is located slightly away from the mitral annular plane. Whether such a distinction is prevalent in human mitral valves is at best speculative, because no such information is yet available from published studies. However, because we used the baseline measurements for each valve as its own control, this anatomic finding did not affect the overall endpoint of our study. Finally, the effect of this altered mitral valve force distribution on the mitral annular and ventricular continuity and ventricular function requires additional investigation in a large animal model, because it remains controversial whether strut chordal cutting diminishes left ventricular function, such as was proposed by Rodriguez and colleagues ${ }^{24}$ and Nielsen and colleagues, ${ }^{25}$ or whether it preserves and possibly enhances the ventricular function by diminishing postinfarction left ventricular remodeling, such as was proposed by Messas and colleagues. ${ }^{9,26}$ Although it is enticing to judge that cutting 2 secondary chordae might not affect global ventricular function, it is important to remember that these chordae, being the 2 load-bearing elements, play a very important role in both the systolic and the diastolic force transfer between the mitral annulus and the apical ventricular wall. A detailed engineering study to measure the valvular-ventricular interactive forces when severing different chordae is thus warranted, not only for the future of mitral repair, but also to better understand chordal sparing valve replacement. The present ex vivo study is unique because it allowed the quantitative assessment of chordal cutting on the changes in the force distribution in the chordae tendineae with various tethering patterns. Although these data suggest that under apical papillary muscle displacement, the changes in chordal forces is minimal, it is well known clinically that the dilated/injured ventricle often remodels after mitral valve repair. ${ }^{27}$ Thus, if chordal cutting is adopted in
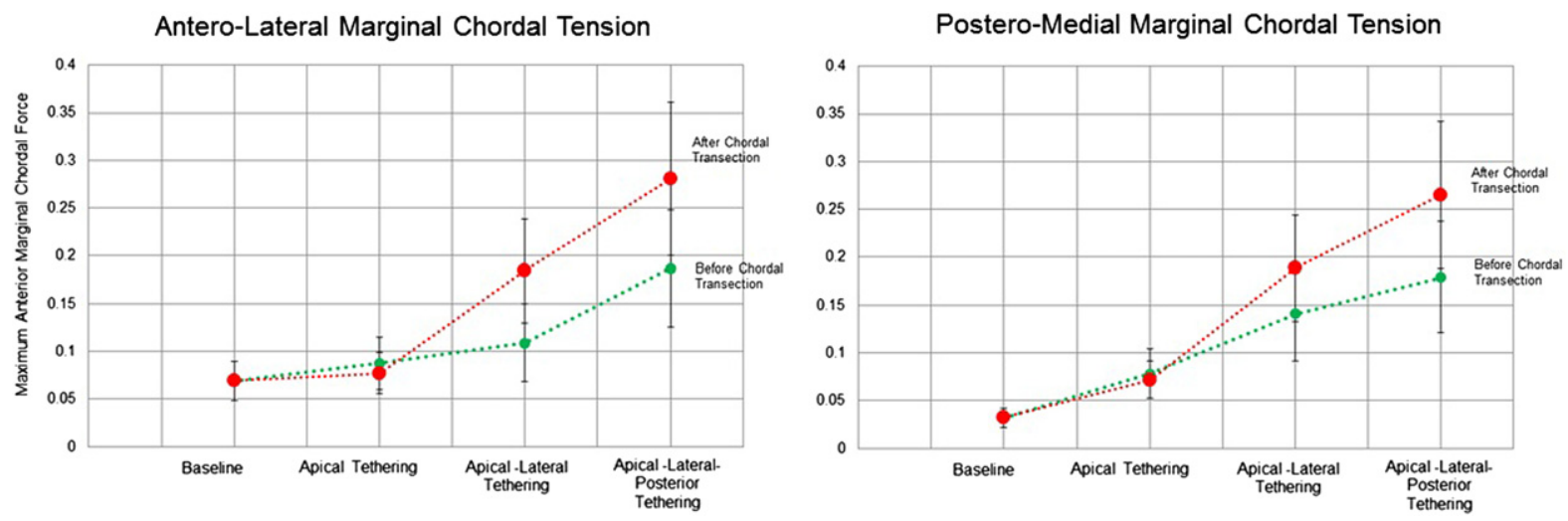

FIGURE 7. Changes in anterior marginal chordal force before and after chordal cutting with progressive change in papillary muscle positions. Slope of increase in marginal chordal force after chordal cutting was significantly greater than before chordal cutting in the case of progressive displacement of papillary muscles after chordal cutting. 
TABLE 1. Continued

\begin{tabular}{|c|c|c|c|c|c|c|c|}
\hline \multicolumn{4}{|c|}{$\begin{array}{l}\text { Average maximum marginal } \\
\text { chordal force after CT cut (n) }\end{array}$} & \multicolumn{2}{|c|}{ Change from baseline (\%) } & \multicolumn{2}{|c|}{ Factor of safety } \\
\hline Left & $P$ value & Right & $P$ value & Left & Right & Left & Right \\
\hline $0.07 \pm 0.03$ & .376 & $0.07 \pm 0.09$ & .85 & 16.7 & 118.7 & 96.14 & 96.14 \\
\hline $0.19 \pm 0.11$ & .025 & $0.198 \pm 0.15$ & .04 & 208.3 & 490.6 & 35.75 & 34.97 \\
\hline $0.30 \pm 0.23$ & .05 & $0.280 \pm 0.21$ & .01 & 366.6 & 728.1 & 23.28 & 24.66 \\
\hline
\end{tabular}

patients with minimal tethering and with apical papillary muscle displacement (eg, that resulting from nonischemic dilated cardiomyopathy causing concentric hypertrophy), it should first be determined whether the ventricle could potentially remodel adversely after the repair. We do recognize that such predictive estimates of ventricular remodeling are currently challenging and, at best, anecdotal. We are hopeful that current research efforts will lead us in that direction. The chordal forces on each valve with the papillary muscle positions as the main effectors are presented in Figure 7, demonstrating that the gradient of increase in the marginal chordal force with evolving papillary muscle displacement was much greater after chordal cutting than before it. It is important that these data should be considered within the ex vivo realm, because clinically it is impossible to predict whether and how much the ventricle would remodel after surgery. Also, other concomitant procedures to the mitral annulus or the leaflets in conjunction with chordal cutting are often performed. Potentially, echocardiographic measurement of the papillary muscle tip to mitral annular plane distance can be used as an indicator for the selection of patients for the chordal cutting procedure. Alternatively, translocation of the transected secondary chordae to another position on the valve or implantation of neochordae to distribute the load could be considered. ${ }^{28,29}$ Additional studies to relate the changes in marginal chordal forces in relation to different mitral valve geometric measurements are currently underway in large animal models of ischemic heart disease.

\section{Study Limitations}

The experimental model used in the present study, although thoroughly validated against large animal models, has some inherent limitations. The lack of atrial and ventricular dynamics could have affected the severity of MR. However, for the scope of our study, this did not overshadow the model's benefits. Mitral annular dynamics were not mimicked, but it was already shown in several animal models that the contractile motion of the annulus is eliminated after annuloplasty. Abnormalities in segmental ventricular wall motion or dyskinesis cannot be simulated in this model to mimic the events involved in adverse remodeling of the ventricle; however, their affect can be easily mimicked by altering the papillary muscle positions.

\section{CONCLUSIONS}

The present quantitative study of the effect of chordal cutting on valve mechanics has demonstrated that significant increases in anterior marginal chordal forces can be expected after strut chordal transection, although in a papillary muscle position-dependent manner. Quantification of the mitral valve annulus to papillary muscle tip distance could be useful in the proper selection of patients for this procedure in the clinical setting.

We thank Holifield Farms for donating the porcine hearts.

\section{References}

1. He S, Fontaine AA, Schwammenthal E, Yoganathan AP, Levine RA. Integrated mechanism for functional mitral regurgitation: Leaflet restriction versus coapting force: In vitro studies. Circulation. 1997;96:1826-34.

2. Komeda M, Glasson JR, Bolger AF, Daughters GT II, MacIsaac A, Oesterle SN. Geometric determinants of ischemic mitral regurgitation. Circulation. 1997; 96(II): 128-33.

3. Anyanwu AC, Adams DH. Ischemic mitral regurgitation: Recent advances. Curr Treat Options Cardiovasc Med. 2008;10:529-37.

4. Calafiore AM, Iacò AL, Contini M, Bivona A, Varone E, Greco P, Scandura S, Di Mauro M. Mitral valve repair for ischemic mitral regurgitation. Angiology. 2008;59:89S-92S.

5. Nesta F, Otsuji Y, Handschumacher MD, Messas E, Leavitt M, Carpentier A, Levine RA, Hung J. Leaflet concavity: A rapid visual clue to the presence and mechanism of functional mitral regurgitation. J Am Soc Echocardiogr. 2003; 16:1301-8.

6. Sai-Sudhakar CB, Vandse R, Armen TA, Bickle KM, Nathan NS. Efficacy of chordal cutting in alleviating ischemic mitral regurgitation: Insights from 3-dimensional echocardiography. J Cardiothorac Surg. 2007;2:39.

7. Messas E, Pouzet B, Touchot B, Guerrero JL, Vlahakes GJ, Desnos M. Efficacy of chordal cutting to relieve chronic persistent ischemic mitral regurgitation. Circulation. 2003;108(Suppl 1):II111-5.

8. Messas E, Guerrero JL, Handschumacher MD, Conrad C, Chow CM, Sullivan S. Chordal cutting: A new therapeutic approach for ischemic mitral regurgitation. Circulation. 2001;104:1958-63.

9. Messas E, Bel A, Szymanski C, Cohen I, Touchot B, Handschumacher MD, Desnos M. Relief of mitral leaflet tethering following chronic myocardial infarction by chordal cutting diminishes left ventricular remodeling. Circ Cardiovasc Imaging. 2010;3:679-86.

10. Borger MA, Murphy PM, Alam A, Fazel S, Maganti M, Armstrong S, et al. Initial results of the chordal-cutting operation for ischemic mitral regurgitation. $J$ Thorac Cardiovasc Surg. 2007; 133:1483-92.

11. Jimenez JH, Soerensen DD, He Z, Ritchie J, Yoganathan AP. Effects of papillary muscle position on chordal force distribution: An in-vitro study. J Heart Valve Dis. 2005; 14:295-302.

12. Ritchie J, Warnock JN, Yoganathan AP. Structural characterization of the chordae tendineae in native porcine mitral valves. Ann Thorac Surg. 2005;80:189-97.

13. He Z, Jowers CW. Effect of mitral valve strut chord cutting on marginal chord tension. J Heart Valve Dis. 2008;17:628-34.

14. He Z, Jowers C. A novel method to measure mitral valve chordal tension. J Biomech Eng. 2009;131:014501. 
15. Chen L, May-Newman K. Effect of strut chordae transection on mitral valve leaflet biomechanics. Ann Biomed Eng. 2006;34:917-26.

16. Nielsen SL, Nygaard H, Fontaine AA, Hasenkam JM, He S, Andersen NT. Chordal force distribution determines systolic mitral leaflet configuration and severity of functional mitral regurgitation. J Am Coll Cardiol. 1999;33:843-53.

17. Sacks MS, Enomoto Y, Graybill JR, Merryman WD, Zeeshan A, Yoganathan AP. In-vivo dynamic deformation of the mitral valve anterior leaflet. Ann Thorac Surg. 2006;82:1369-77.

18. Sedransk KL, Grande-Allen KJ, Vesely I. Failure mechanics of mitral valve chordae tendineae. J Heart Valve Dis. 2002;11:644-50.

19. Salisbury PF, Cross CE, Rieben PA. Chorda tendinea tension. Am J Physiol. 1963;205:385-92.

20. Nielsen SL, Hansen SB, Nielsen KO, Nygaard H, Paulsen PK, Hasenkam JM. Imbalanced chordal force distribution causes acute ischemic mitral regurgitation: Mechanistic insights from chordae tendineae force measurements in pigs. $J$ Thorac Cardiovasc Surg. 2005;129:525-31.

21. Nielsen SL, Nygaard H, Mandrup L, Fontaine AA, Hasenkam JM, He S. Mechanism of incomplete mitral leaflet coaptation-Interaction of chordal restraint and changes in mitral leaflet coaptation geometry. Insight from in vitro validation of the premise of force equilibrium. J Biomech Eng. 2002;124: 596-608.

22. Glasson JR, Komeda M, Daughters GT, Bolger AF, Karlsson MO, Foppiano LE. Early systolic mitral leaflet "loitering" during acute ischemic mitral regurgitation. J Thorac Cardiovasc Surg. 1998;116:193-205.
23. Pedersen LG, Zhao J, Yang J, Thomsen PD, Gregersen H, Hasenkam JM. Increased expression of endothelin B receptor in static stretch exposed porcine mitral valve leaflets. Res Vet Sci. 2007;82:232-8.

24. Rodriguez F, Langer F, Harrington KB, Tibayan FA, Zasio MK, Cheng A. Importance of mitral valve second-order chordae for left ventricular geometry, wall thickening mechanics, and global systolic function. Circulation. 2004;110: II115-22.

25. Nielsen SL, Timek TA, Green GR, Dagum P, Daughters GT, Hasenkam JM. Influence of anterior mitral leaflet second-order chordae tendineae on left ventricular systolic function. Circulation. 2003;108:486-91.

26. Messas E, Yosefy C, Chaput M, Guerrero JL, Sullivan S, Menasché P. Chorda cutting does not adversely affect left ventricle contractile function. Circulation. 2006;114:I524-8.

27. Pfeffer JM, Pfeffer MA, Fletcher PJ, Braunwald E. Progressive ventricular remodeling in rat with myocardial infarction. Am J Physiol. 1991;260 H1406-14.

28. Masuyama S, Marui A, Shimamoto T, Komeda M. Chordal translocation: Secondary chordal cutting in conjunction with artificial chordae for preserving valvular-ventricular interaction in the treatment of functional mitral regurgitation. J Heart Valve Dis. 2009;18:142-6.

29. Masuyama S, Marui A, Shimamoto T, Nonaka M, Tsukiji M, Watanabe N. Chordal translocation for ischemic mitral regurgitation may ameliorate tethering of the posterior and anterior mitral leaflets. J Thorac Cardiovasc Surg. 2008;136 868-75. 


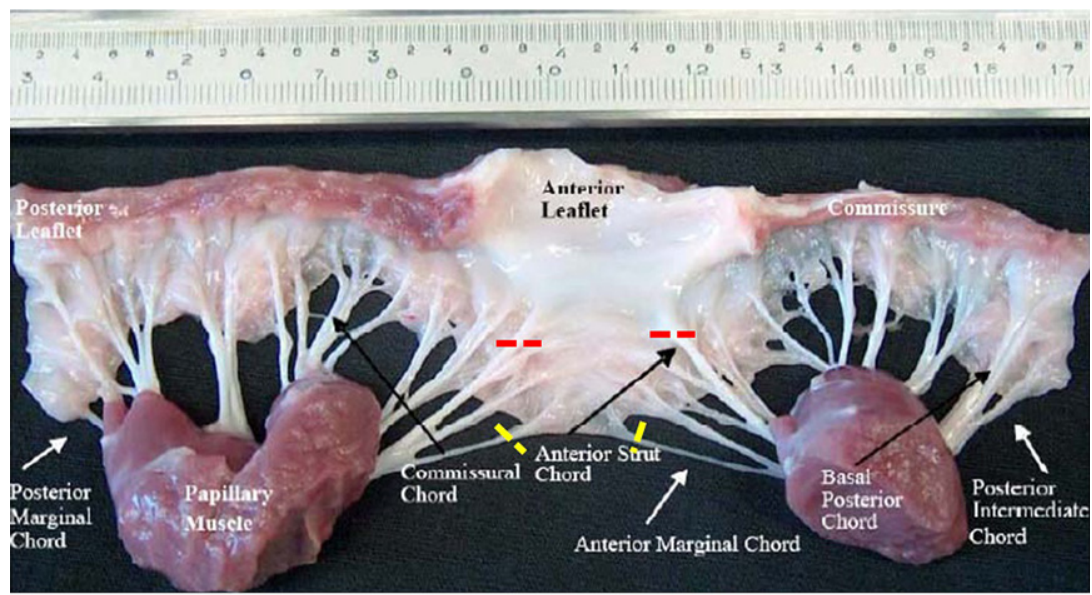

Location where the strut chordae was transected on the postero-medial papillary muscle

$=$ Location where the force transducers were mounted on the marginal chordae

FIGURE E1. Photograph of a porcine mitral valve depicting the various chordae tendineae classified based on their insertion zone in the mitral leaflet. The red dotted lines depict the location of the strut chordal transection and the yellow dotted lines depict the location where the force transducers are mounted.

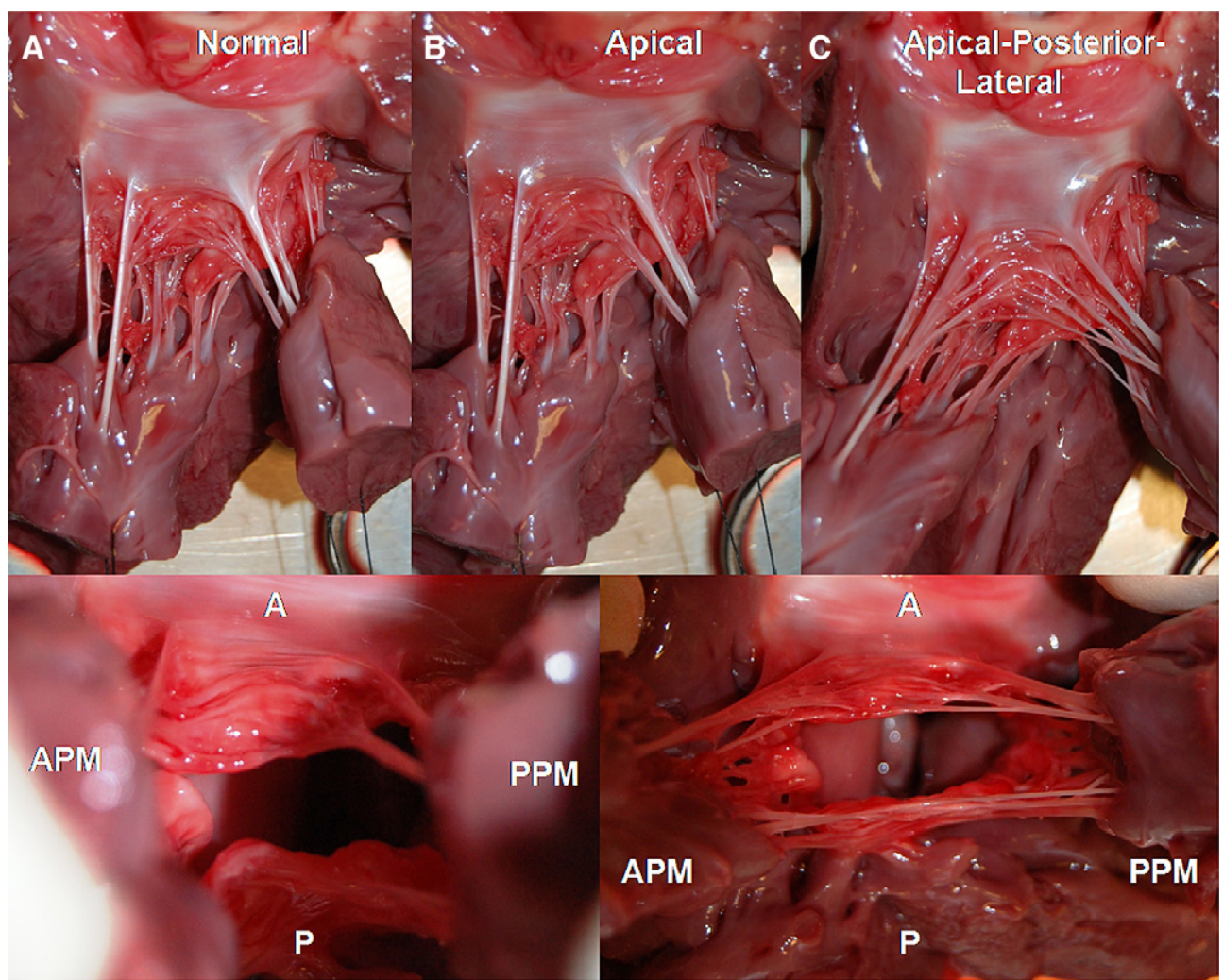

FIGURE E2. Native porcine valve depicting increase in free edge tethering even under diastolic conditions, with lateral and posterior displacement of papillary muscles. Such tethering not evident with just apical displacement. $A=$ Anterior; $A P M=$ apical papillary muscle; $P=$ posterior; $P P M=$ posterior papillary muscle. 

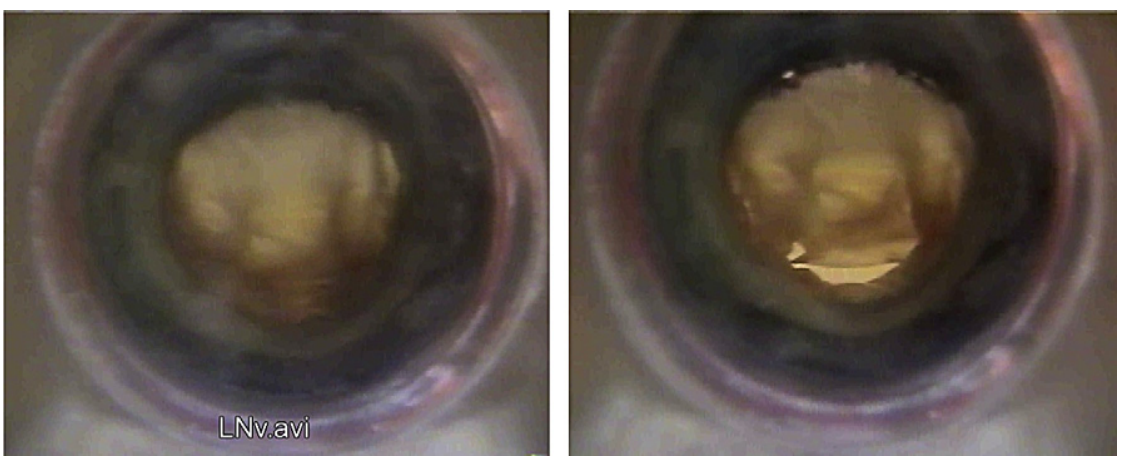

FIGURE E3. Left, Valve from experimental setup in which chordal cutting relieved leaflet tethering and restored normal leaflet coaptation with apical papillary muscle displacement only. Right, Regurgitant orifice persisted even after repair procedure with apical posterior and lateral displacement of muscles. 